\title{
Restoration of osteogenic differentiation by overexpression of cannabinoid receptor 2 in bone marrow mesenchymal stem cells isolated from osteoporotic patients
}

\author{
BANGJUN WANG, KAI LIAN, JUN LI and GANG MEI \\ Department of Orthopaedic Surgery, Xiangyang Central Hospital, The Affiliated Hospital of \\ Hubei University of Arts and Science, Xiangyang, Hubei 441021, P.R. China
}

Received June 4, 2016; Accepted April 11, 2017

DOI: 10.3892/etm.2017.5369

\begin{abstract}
Cannabinoid receptor 2 (CNR2) has a critical role in osteogenic differentiation of bone marrow mesenchymal stem cells (BMSCs). CNR2 expression was found to be downregulated in osteoporotic patients. The present study aimed to investigate the functionality of CNR2 in restoring osteogenic differentiation and mineralization of BMSCs isolated from osteoporotic patients. CNR2 was overexpressed in osteoporotic BMSCs by a lentivirus. Alkaline phosphatase (ALP) activity staining and alizarin red $\mathrm{S}$ staining were performed to examine the osteogenic differentiation of osteoporotic BMSCs. Reverse-transcription quantitative polymerase chain reaction analysis was performed to examine the expression of osteogenic genes in BMSCs. Western blot analysis was used to study the activation of p38 mitogen-activated protein kinase (MAPK) during osteogenic differentiation of osteoporotic BMSCs after lentivirus-mediated overexpression of CNR2. The results demonstrated that overexpression of CNR2 in osteoporotic BMSCs increased ALP activity, promoted expression of osteogenic genes and enhanced deposition of mineralized extracellular matrix. In addition, phosphorylation of p38 MAPK was found to be increased by overexpression of CNR2. In conclusion, the present study indicated that restoration of CNR2 recovered the osteogenic differentiation of BMSCs isolated from osteoporotic patients. This finding may provide a novel strategy for a treatment approach for osteoporosis.
\end{abstract}

Correspondence to: Dr Kai Lian, Department of Orthopaedic Surgery, Xiangyang Central Hospital, The Affiliated Hospital of Hubei University of Arts and Science, 136 Jinzhou Street, Xiangyang, Hubei 441021, P.R. China

E-mail:kailian49@sina.com

Key words: cannabinoid receptor 2, overexpression, p38 mitogen activated protein kinase, lentiviral transduction, osteoporosis

\section{Introduction}

Osteoporosis (OP) is considered as one of the most common chronic skeletal diseases in the aged population of modern society. OP is a disorder of the skeletal system that leads to decreased bone density and increased fracture risk due to an imbalance in bone matrix turnover. According to the World Health Organization, patients diagnosed with OP demonstrate significantly decreased bone mineral density (BMD) compared with that of sex-matched adults (1). Osteoporotic fractures occur so frequently as to affect 1 in 3 women and 1 in 5 men over 50 years of age (2). The increasing number of osteoporotic fractures not only places a heavy economic burden on the medical care system but also leads to chronic pain, deformity, depression, disability and even death of patients.

Cannabinoid receptors (CNRs) are members of the G-protein coupled receptors superfamily and have a major role in the pathophysiology of a wide range of chronic diseases. CNR2 (also abbreviated as $\mathrm{CB}_{2}$ receptor), is considered to be a promising drug target for the treatment of a number of diseases, including osteoporosis (3). Due to the lack of CNR2 in the central neural system (CNS), CNR2 receptor-targeting drugs usually show only few adverse effects on the CNS (4). Great progress has been made in the past few decades in the identification of novel ligands and optimization of the binding efficiency to the CNR2 (5). However, not until recently, endogenous cannabinoids and corresponding receptors were found to be involved in the regulation of osteoblast differentiation and bone formation (6,7). For instance, $\mathrm{CB}_{1 / 2}$ receptor agonist $\mathrm{CP} 55,940$ and CNR2-selective agonist HU 308 have been shown to stimulate the early differentiation of bone marrow-derived osteoblast precursors and enhance bone nodule formation in osteoblast cultures in vitro (8). Consistent with these discoveries, a previous study by our group found that activation of CNR2 by chemical ligand UR-144 enhanced osteogenic differentiation of bone marrow-derived mesenchymal stem cells (BMSCs) (9), whereas treatment with the CNR1 antagonist AM 251 suppressed osteoblast number and function (10). Therefore, it appears that cannabinoid signaling functions differently in terms of different cannabinoid receptors, but its role in osteoporosis has remained elusive. 
Previous studies have revealed the loss of CNR2 expression in the bone marrow of osteoporotic patients (9). The present study hypothesized that lentiviral vector-mediated overexpression of CNR2 reverses the osteogenic differentiation and mineralization ability of BMSCs isolated from osteoporotic patients. To test this hypothesis, patient-derived BMSCs were transfected with CNR2-expressing lentiviral vector, and their osteogenic differentiation ability and associated cell signaling were tested. Regulation of CNR2 in osteoporosis patient-derived BMSCs may provide a promising therapeutic strategy, which transforms local stem cells from a disease state to a reparative state.

\section{Materials and methods}

Cell culture and expansion. Bone marrow biopsies were obtained from patients who underwent bone marrow examinations in October 2015. Patients provided informed consent before bone marrow collection. The protocol for human tissue preparation was approved by the Medical Ethical Committee of Xiangyang Central Hospital (Xiangyang, China). MSCs were isolated from bone marrows of osteoporotic patients as well as healthy donors as described previously (11). In brief, total bone marrow was plated at a density of 50,000 cells $/ \mathrm{cm}^{2}$ in culture flasks in MSC proliferation medium $[\alpha$-modified Eagle's medium ( $\alpha$-MEM) supplemented with $10 \%$ fetal bovine serum (FBS), $1 \%$ L-glutamine, $0.2 \mathrm{mM}$ ascorbic acid, $100 \mathrm{U} / \mathrm{ml}$ penicillin, $10 \mu \mathrm{g} / \mathrm{ml}$ streptomycin and $1 \mathrm{ng} / \mathrm{ml}$ basic fibroblast growth factor], plus $1 \%$ heparin. The medium was changed every 3-4 days until confluence. BMSCs from four healthy and four osteoporotic patients were used in this study. All reagents used for cell culture were purchased from Invitrogen (Thermo Fisher Scientific, Inc., Waltham, MA, USA) and chemicals were purchased from Sigma-Aldrich (Merck KGaA, Darmstadt, Germany), unless otherwise specified.

Osteogenic differentiation. Osteogenic differentiation was induced by treating MSCs with osteogenic medium containing Dulbecco's modified Eagle's medium plus $10 \%$ FBS, $0.1 \mathrm{nM}$ dexamethasone, $10 \mathrm{mM} \beta$-glycerophosphate, $0.01 \mu \mathrm{M} 1,25$-dihydroxy vitamin D3 and $50 \mu \mathrm{M}$ ascorbic acid in $\alpha$-MEM (12).

Alizarin red staining. After 3 weeks of culture in osteogenic medium supplemented with $10 \mathrm{nM} \mathrm{CNR} 2$ receptor agonist UR-144, MSCs were fixed with $10 \%$ formalin. After rinsing in PBS, cells were incubated with $40 \mathrm{mM}$ alizarin red $\mathrm{S}(\mathrm{pH} 4.2)$ for $10 \mathrm{~min}$ with agitation. Cells were then rinsed 5 times with water, followed by washing with PBS for 15 min to reduce non-specific staining. The stained nodules were observed and recorded by using a phase contract microscope.

Alkaline phosphatase (ALP) activity staining. Cytochemical analysis with 5-bromo-4-chloro-3-indolyl phosphate (BCIP) and nitro blue tetrazolium chloride (NBT) was used for the staining for ALP. MSCs were first fixed in $10 \%$ formalin and incubated with $300 \mu \mathrm{l}$ BCIP/NBT premixed solution (Sigma-Aldrich; Merck KGaA) for 8-10 min at room temperature. Cells were then rinsed with water, dried and examined by phase contract microscopy.

RNA isolation and reverse-transcription quantitative polymerase chain reaction $(R T-q P C R)$. Total RNA was isolated from BMSCs with the QIAamp DNA Mini kit (Qiagen, Hilden, Germany). One microgram of total RNA was reverse-transcribed into complementary (c)DNA using the iScript cDNA Synthesis kit (Bio-Rad Laboratories, Inc., Hercules, CA, USA). The cDNA samples were amplified with a Pfu PCR kit (Tiangen, Beijing, China) with specific primers listed in Table I. All PCR products then underwent electrophoresis on a 2\% agarose gel. Quantitative PCR was performed on cDNA samples by using the iQ SYBR Green Supermix (Bio-Rad, Laboratories, Inc.). PCR was performed on a MyiQ2 Two-Color Real-Time PCR Detection System (Bio-Rad Laboratories, Inc.) under the following conditions: cDNA was pre-heated for $15 \mathrm{~min}$ at $95^{\circ} \mathrm{C}$ and denatured for $5 \mathrm{~min}$ at $95^{\circ} \mathrm{C}$, followed by 45 cycles consisting of $15 \mathrm{sec}$ at $95^{\circ} \mathrm{C}, 15 \mathrm{sec}$ at $60^{\circ} \mathrm{C}$ and $30 \mathrm{sec}$ at $72^{\circ} \mathrm{C}$. For each reaction, a melting curve was generated to confirm the binding specificity of the primers. Calculation of relative expression was performed using iQ5 optical system software (version 2.0; Bio-Rad Laboratories, Inc.) using the $\Delta \Delta \mathrm{Cq}$ method (13). GAPDH was used as housekeeping gene for normalization.

Overexpression of CNR2 by lentiviral vector in MSCs. Cloning of CNR2 and lentiviral vectors were prepared by Hanbio Biotechnology (Shanghai, China) to overexpress CNR2 in target cells. In brief, the cDNA of CNR2 was amplified with specific primers from a cDNA library and sub-cloned into a $\mathrm{T}$ vector. The coding sequence of CNR2 was then cloned into lentiviral vectors ( $\mathrm{pLVX}$-IRES-ZsGreen1) that expressed GFP and puromycin-resistant proteins for screening. Lentiviral supernatants (Lenti-CNR2) were packaged by triple transfection of HEK293T cells (Fig. 1). MSCs were infected by adding virus-containing supernatants to the culture medium. Transduced cells were selected by addition of puromycin $(1 \mu \mathrm{g} / \mathrm{ml})$ at 2 days after infection for 1 week. Transduction was verified by visual examination for cells expressing green fluorescent protein (GFP) under a fluorescent microscope. Cells infected with lentivirus containing empty vectors (Lenti-GFP) were used as controls.

Immunofluorescent staining. MSCs with or without viral infection were seeded on glass cover slips in six-well plates $24 \mathrm{~h}$ prior to staining. Cells were washed with PBS, fixed in $4 \%$ paraformaldehyde for $30 \mathrm{~min}$ at room temperature and then permeabilized with $1 \%$ Triton-X 100 prior to being blocked in $1 \%$ bovine serum albumin for $15 \mathrm{~min}$ at room temperature. Slips were subsequently incubated overnight at $4{ }^{\circ} \mathrm{C}$ with rabbit polyclonal primary antibodies against CNR2 receptor with a dilution of 1:100 (cat. no. ab3561, Abcam, Cambridge, MA). Sequentially, slides were incubated with secondary antibodies conjugated to Alexa 594 with a dilution of 1:500 for $1 \mathrm{~h}$ at room temperature (cat. no. A-11037, Invitrogen; Thermo Fisher Scientific, Inc.) and cell nuclei were counterstained with 4,6-diamidino-2-phenylindole (Molecular Probes, Eugene, OR, USA). After rinsing with PBS, cells were examined and 
Table I. Sequences of primers used for polymerase chain reaction.

\begin{tabular}{|c|c|c|c|}
\hline Gene name & NCBI Gene ID & Sequence $\left(5^{\prime}-3^{\prime}\right)$ & Length of amplicon (bp) \\
\hline Cannabinoid receptor 2 & 1,269 & $\begin{array}{l}\text { Forward, AGCCCTCATACCTGTTCATTGG; } \\
\text { Reverse, GTGAAGGTCATAGTCACGCTG }\end{array}$ & 154 \\
\hline Runt-related transcription factor 2 & 860 & $\begin{array}{l}\text { Forward, TGGTTACTGTCATGGCGGGTA; } \\
\text { Reverse, TCTCAGATCGTTGAACCTTGCTA }\end{array}$ & 101 \\
\hline Osterix & & $\begin{array}{l}\text { Forward, CCTCTGCGGGACTCAACAAC; } \\
\text { Reverse, AGCCCATTAGTGCTTGTAAAGG }\end{array}$ & 128 \\
\hline Integrin-binding sialoprotein & 3,381 & $\begin{array}{l}\text { Forward, CACTGGAGCCAATGCAGAAGA; } \\
\text { Reverse, TGGTGGGGTTGTAGGTTCAAA }\end{array}$ & 106 \\
\hline Osteocalcin & 632 & $\begin{array}{l}\text { Forward, CACTCCTCGCCCTATTGGC; } \\
\text { Reverse, CCCTCCTGCTTGGACACAAAG }\end{array}$ & 112 \\
\hline Secreted phosphoprotein 1 & 6,696 & $\begin{array}{l}\text { Forward, GAAGTTTCGCAGACCTGACAT; } \\
\text { Reverse, GTATGCACCATTCAАCTCCTCG }\end{array}$ & 91 \\
\hline GAPDH & 2,597 & $\begin{array}{l}\text { Forward, CTGGGCTACACTGAGCACC; } \\
\text { Reverse, AAGTGGTCGTTGAGGGCAATG }\end{array}$ & 101 \\
\hline
\end{tabular}

NCBI, national center for biotechnology information.

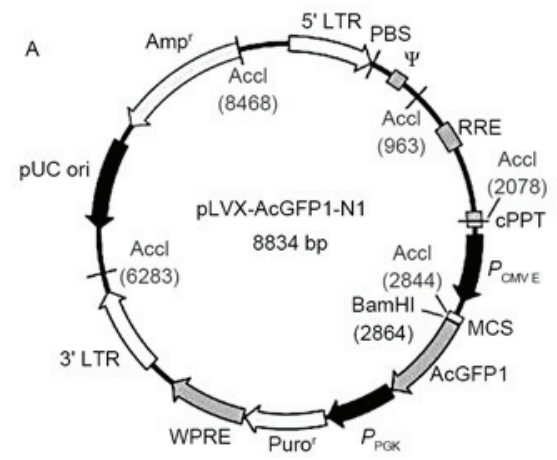

B

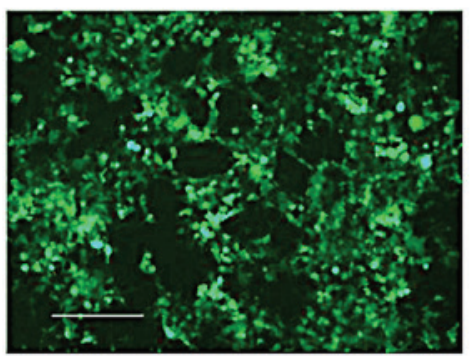

C

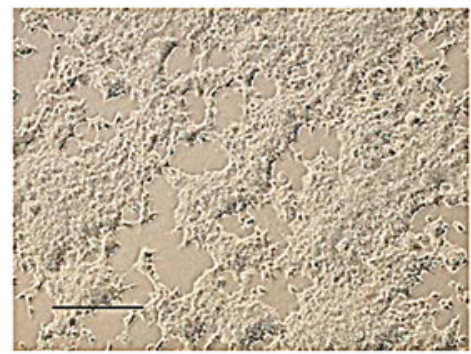

Figure 1. Construction of letiviral vector and packaging of letivirus. (A) Map of shuttle vector. (B) Transfected T293 cells express GFP. (C) Phase-contrast image of transfected T293 cells (scale bars, $100 \mu \mathrm{m}$ ). GFP, green fluorescence protein.

imaged using a DMi 6000 B fluorescent microscope (Leica Microsystems, Wetzlar, Germany).

Total protein isolation and western blot analysis. Prior to lysing cells, cell layers were washed three times with PBS. Total protein was extracted by RIPA Lysis and Extraction buffer (Thermo Fisher Scientific, Inc.), followed by centrifugation at $13,000 \mathrm{x} \mathrm{g}$ for $15 \mathrm{~min}$ to remove cell debris. The protein concentration was measured and normalized using a bicinchoninic acid kit (Pierce; Thermo Fisher Scientific, Inc.). Twenty-five micrograms of total protein from each sample was loaded and separated by $15 \%$ SDS-PAGE gel, then bands were transferred to PVDF membranes. Then, membranes were incubated with polyclonal rabbit anti-CNR2 antibody (cat. no. ab3561, Abcam) or anti-phospho-p38 mitogen-activated protein kinase (MAPK) (Thr180/Tyr182) antibody (cat. no. 4511S, Cell Signaling Technology, Inc., Danvers, MA, USA) with dilution of 1:1,000 at $4^{\circ} \mathrm{C}$ overnight. After washing, membranes were incubated with HRP-conjugated secondary antibodies (cat. no. 31460, Thermo Fisher Scientific, Inc.) with dilution of 1:1,000 for $1 \mathrm{~h}$ at room temperature.
Immunocomplexes were visualized using enhanced chemiluminescence reagent (Thermo Fisher Scientific, Inc.). Band intensities were analyzed by Image J software (NIH, Bethesda, MD, USA).

Statistical analysis. Data are presented as the mean \pm standard deviation. Student's t-tests for paired samples were performed to determine statistical significance between two groups, with GraphPad Prism 7.0 (GraphPad Software, Inc. La Jolla, CA, USA). $\mathrm{P}<0.05$ was considered to indicate a statistically significant difference.

\section{Results}

CNR2 is expressed in normal BMSCs, while absent in osteoporotic BMSCs. Previous studies have revealed the loss of CNR2s expression in the bone marrow of osteoporotic patients. However, MSC-specific expression patterns of CNR2 in bone marrow has remained to be fully elucidated. The present study assessed the expression of CNR2 in cultured BMSCs isolated from healthy individuals and osteoporotic 
A

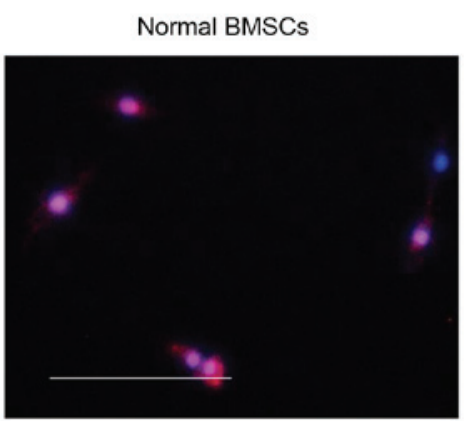

B

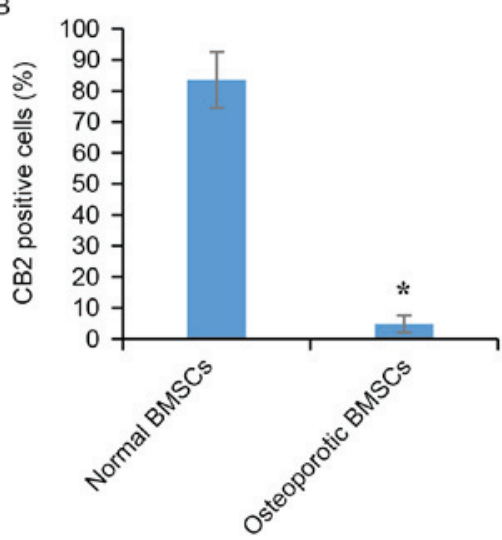

Osteoporotic BMSCs

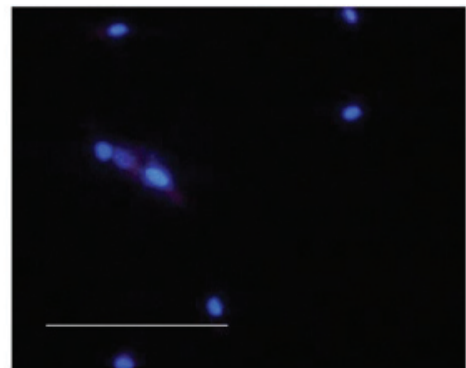

C

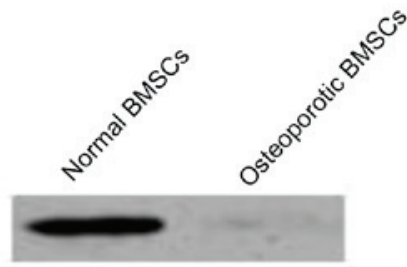

$\beta$-actin

Figure 2. Expression of CNR2 receptor in normal and osteoporotic BMSCs. (A) Immunofluorescent staining of CB2 receptors present on normal BMSCs but not on osteoporotic BMSCs (scale bars, $100 \mu \mathrm{m}$ ). (B) Quantification of immunofluorescent images provided the ratio of CNR2-positive cells in normal and osteoporotic BMSCs. (C) Western blot confirmed that CNR2 receptor was mainly expressed on normal but not on osteoporotic BMSCs. "P<0.05 vs. normal BMSCs. BMSCs, bone marrow mesenchymal stem cells; CNR2, cannabinoid receptor 2.

A

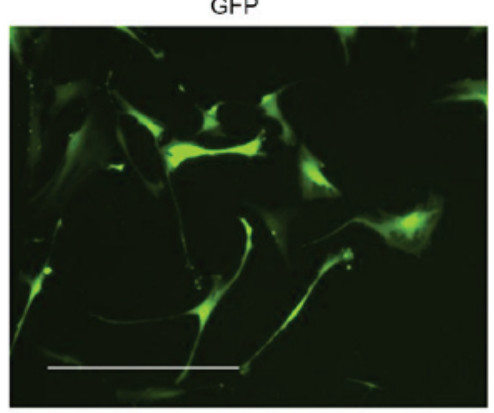

B

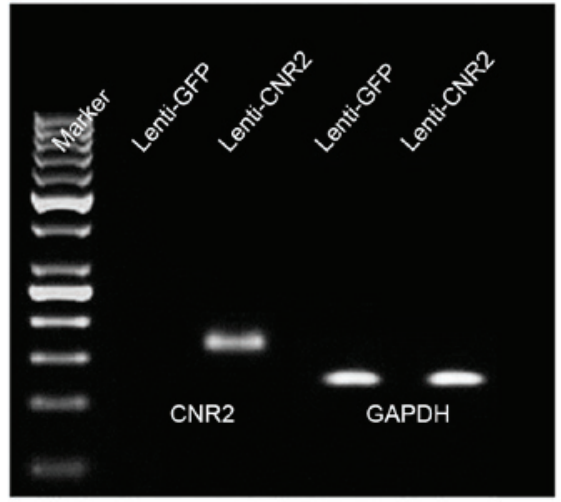

$\mathrm{CB}_{2}$ receptor

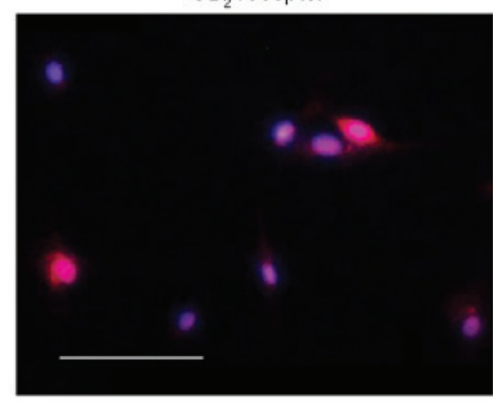

C

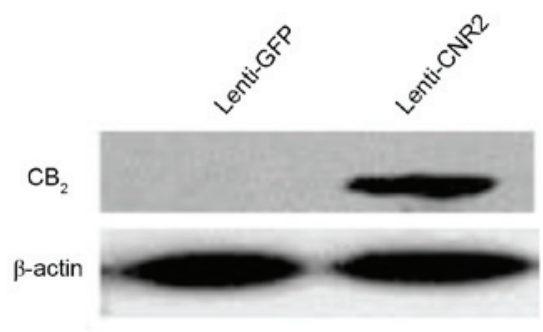

Figure 3. Overexpression of CNR2 gene in osteoporotic BMSCs. (A) $48 \mathrm{~h}$ after lentiviral transfection, osteoporotic BMSCs expressed GFP, which is an indication of successful transfection (scale bars, $50 \mu \mathrm{m}$ ). Immunofluorescent staining also revealed that cells expressed CNR2 receptor. (B) Reverse-transcription quantitative polymerase chain reaction analysis and (C) western blot was also performed $48 \mathrm{~h}$ after infection, confirming the overexpression of CNR2 genes in osteoporotic BMSCs. BMSCs, bone marrow mesenchymal stem cells; CNR2, cannabinoid receptor 2; GFP, green fluorescence protein; Lenti, lentivirus. 
Normal BMSCs

A

B

$\mathrm{C}$

ALP quantification

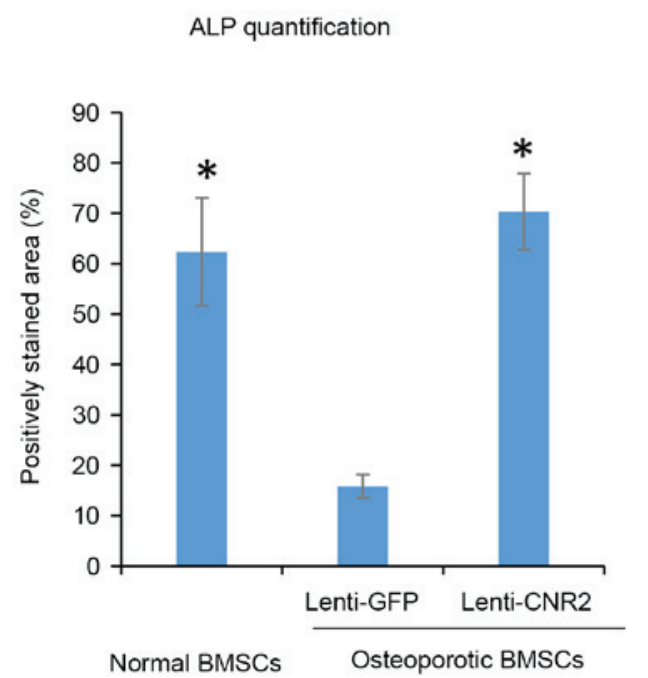

Osteoporotic BMSCs

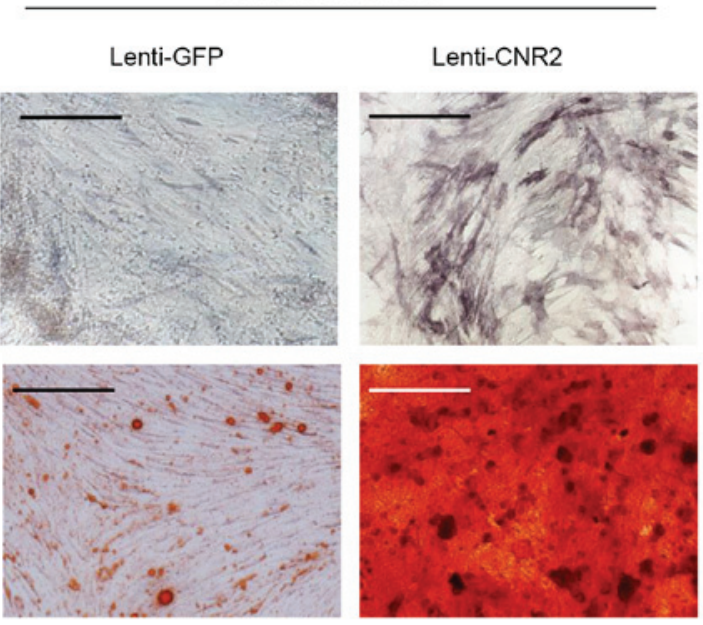

D

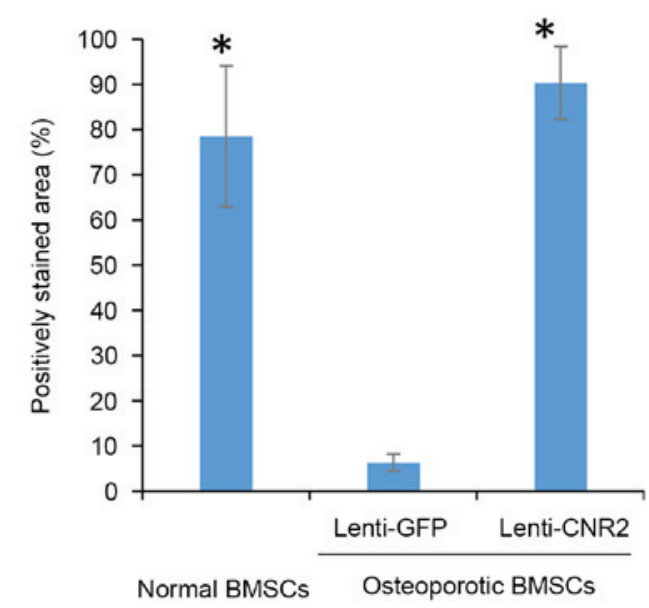

Figure 4. Overexpression of CNR2 gene increases mineralization of osteoporotic BMSCs. (A) Osteoporotic BMSCs infected with Lenti-CNR2 had a higher alkaline phosphatase activity at 2 weeks after culture in osteogenic medium. (B) Alizarin Red S staining indicated that overexpression of CNR2 gene promoted mineralization in osteoporotic BMSCs (scale bars, $100 \mu \mathrm{m}$ ). (C) Quantification of ALP staining. (D) Quantification of Alizarin Red S staining. "P<0.05 vs. Lenti-GFP transfected osteoporotic BMSCs. BMSCs, bone marrow mesenchymal stem cells; CNR2, cannabinoid receptor 2; GFP, green fluorescence protein; Lenti, lentivirus; ALP, alkaline phosphatase.

patients. Immunofluorescent staining showed that CNR2 was only expressed in normal BMSCs but absent in osteoporotic BMSCs (Fig. 2A). Quantification of immunofluorescent images indicated that CNR2-expressing cells accounted for $>80 \%$ of the overall BMSC population in healthy individuals but $<5 \%$ in osteoporotic BMSCs (Fig. 2B). Western blot analysis also demonstrated significantly lower protein levels of CNR2 in BMSCs from osteoporotic patients compared to in BMSCs from healthy individuals (Fig. 2C).

CNR2 expression in osteoporotic BMSCs may be restored by lentiviral infection. To overexpress CNR2 in osteoporotic BMSCs, the coding sequence of CNR2 was cloned into lentiviral vectors (Fig. 1), which were transfected into BMSCs. Successful transduction was identified by GFP expression after screening with antibiotics. After puromycin treatment, all live cells expressed GFP, indicating a homogenously transfected cell population after selection (Fig. 3A). Immunofluorescent staining, RT-qPCR and western blot analysis were performed to assess the expression of CNR2. As shown in Fig. 3A, CNR2 was present in BMSCs transduced with Lenti-CNR2, but absent in BMSCs transduced with control virus (Lenti-GFP). RT-qPCR and western blot confirmed the expression of CNR2 at the mRNA and protein level (Fig. 3B and C).

Overexpression of CNR2 promotes osteogenic differentiation of BMSCs isolated from osteoporotic patients. To test the possible influence of CNR2 overexpression on the mineralization of osteoporotic BMSCs, cells transfected with Lenti-CNR2 were cultured in osteogenic medium to induce osteogenic differentiation. BMSCs isolated from healthy individuals were used as positive controls, while osteoporotic BMSCs infected with Lenti-GFP were used as a negative control. All groups of cells were cultured in osteogenic medium for 2 weeks for ALP activity assay and 3 weeks for the mineralization assay. As presented in Fig. 4A, osteoporotic BMSCs infected with 

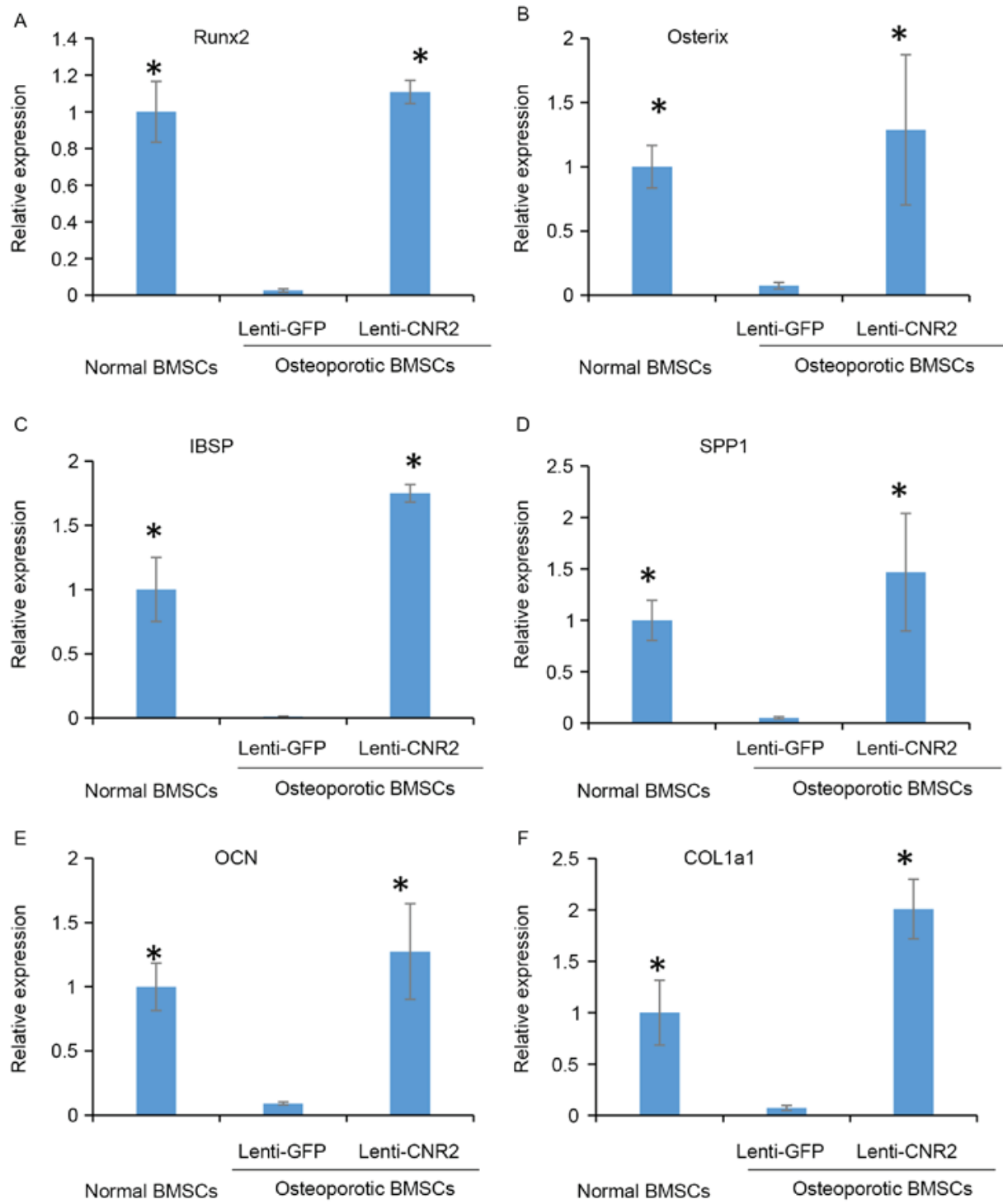

Figure 5. Overexpression of CNR2 gene increases expression of osteogenic genes in osteoporotic BMSCs. Expression of (A) Runx2 (B) Osterix (C) IBSP (D) SPP1 (E) OCN and (F) COL 1a1 were detected by reverse-transcription quantitative polymerase chain reaction analysis at three weeks after osteogenic induction. " $\mathrm{P}<0.05$ vs. Lenti-GFP transfected osteoporotic BMSCs. BMSCs, bone marrow mesenchymal stem cells; CNR2, cannabinoid receptor 2; GFP, green fluorescence protein; Lenti, lentivirus; Runx2, runt-related transcription factor 2; IBSP, integrin-binding sialoprotein; SPP1, secreted phosphoprotein 1; OCN, osteocalcin; COL1a1, collagen type I $\alpha 1$ chain.

Lenti-CNR2 exhibited evidently increased ALP activity compared to those infected with Lenti-GFP only, suggesting the rescue of ALP activity by lentivirus-mediated CNR2 overexpression. Alizarin red S staining revealed a significantly higher amount of mineral deposition in extracellular matrix secreted by CNR2-overexpressing BMSCs (Fig. 4B). Quantification of staining images (Fig. 4C and D) confirmed that ALP activity and calcium accumulation were higher in osteoporotic BMSCs infected with Lenti-CNR2 than in those transfected with Lenti-GFP. These findings were also consistent with the difference in expression levels of genes associated with mineralization among groups, including runt-related transcription factor 2, integrin-binding sialoprotein, secreted phosphoprotein 1, osteocalcin and collagen type I $\alpha 1$ chain (Fig. 5).

Overexpression of CNR2 increases phosphorylation of p38 MAPK. Since activation of p38 MAPK is known to be
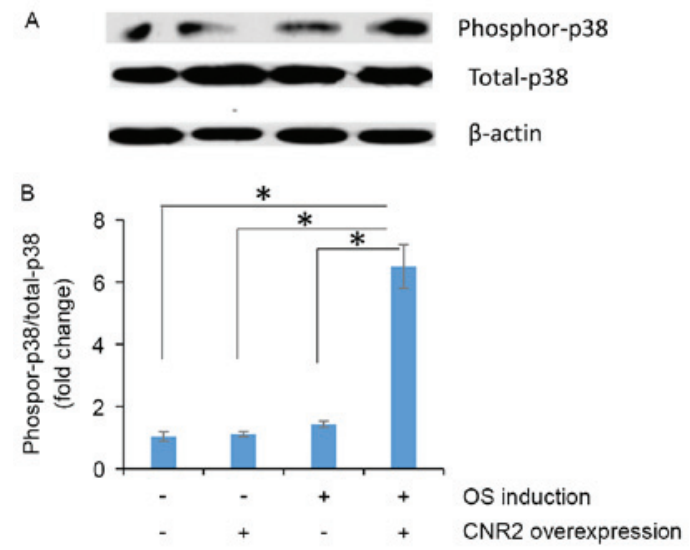

Figure 6. CNR2 gene overexpression increases phosphorylation of $\mathrm{p} 38$ MAPK. (A) Western blot analysis was performed at $24 \mathrm{~h}$ after osteogenic induction. (B) Blot images were quantified to compare the amount of phosphorylated p38 MAPK between several groups. "P<0.05. OS, osteogenic; MAPK, mitogen-activated protein kinase; CNR2, cannabinoid receptor 2. 
involved in CNR2-mediated mineralization, the present study examined the phosphorylation of p38 MAPK in the different groups by western blot analysis. As displayed in Fig. 6A, overexpression of CNR2 increased the phosphorylation of p38 MAPK in osteoporotic BMSCs. Quantification of western blot images confirmed significantly higher phosphorylation level of p38 MAPK in BMSCs transfected with Lenti-CNR2 than that in BMSCs transfected with Lenti-GFP $(\mathrm{P}<0.001$; Fig. 6B).

\section{Discussion}

The current US Food and Drug Administration-approved pharmacological therapies for osteoporosis prevention and/or treatment are bisphosphonates, calcitonin and hormone therapy, including estrogens, parathyroid hormone and estrogen agonists/antagonists (14). The mechanism of bisphosphonate-induced bone mineralization involves the inhibition of osteoclasts by bisphosphonates (15). Orally administered bisphosphonates reduced the vertebral and non-vertebral fracture risk in $20-50 \%$ of subjects relative to the placebo group, while it was also associated with gastrointestinal toxicity and adverse side effects (16). Hormone therapy reduced the fracture risk in $30-40 \%$ of subjects relative to the placebo group (17); however, continuous use is required to achieve efficacy, and hormone therapy may confer beneficial effects on bone and also stimulation of other tissues (18). Long-term therapy may increase the risk of adverse health outcomes, such as stroke and breast cancer in women (19). An enhanced understanding of the pathogenesis of osteoporosis may provide more precise cellular targets.

Stem cells have an important role in tissue hemostasis and repair (20,21). However, aging and diseases may cause a loss of function of stem cells $(22,23)$. A previous study by our group showed that bone marrow CNR2 levels in osteoporotic patients were significantly reduced (9). In the present study, overexpression of CNR2 was shown to restore the osteogenic differentiation and mineralization of BMSCs isolated from osteoporotic patients. Thus, it is possible that modification of stem cells from a disease state into a reparative state has a therapeutic effect.

The present study found that osteoporotic BMSCs transfected with Lenti-CNR2 exhibited higher ALP activity, higher expression levels of osteogenic genes and increased mineral deposition in the extracellular matrix than BMSCs transfected with Lenti-GFP. It was further demonstrated that p38 MAPK phosphorylation was increased by CNR2 overexpression. Human CNR1/2 may be activated by endogenous release of 'endocannabinoids' or exogenous administration of drugs to ameliorate and cure diseases induced by CNR1/2 inactivation. A number of medicines that activate CNR1/2 are now used in clinical practice, including Cesamet (nabilone), Marinol \{dronabinol; $\Delta[9]$-tetrahydrocannabinol $[\Delta(9)-\mathrm{THC}]\}$ and Sativex [ $\Delta(9)$-THC with cannabidiol]. Particular research interests have focused on the development of CNR2-selective medicines with little activating effect on CNR1, due to the fact that adverse effects induced by mixed CNR $1 / 2$ receptor agonists primarily result from CNR1 rather than from CNR2 activation, and that CNR2-selective agonists have demonstrated greater potential for therapeutic applications $(24,25)$. The present study provided a specific strategy for treating osteoporosis, as bone marrow stem cells regained their osteogenesis ability following restoration of CNR2. It is important for future studies to assess whether restoration of CNR2 inhibits osteoporosis-associated bone remodeling in vivo by using animal models.

In conclusion, the present study supported that CNR2 in BMSCs is a therapeutic target of osteoporosis and overexpression of CNR2 restores the osteogenic differentiation ability of BMSCs from osteoporotic patients. The present study provided a cellular therapeutic target for osteoporosis, which may lead to the development of more effective drugs in the near future.

\section{Acknowledgements}

This study was funded by the National Natural Science Foundation of China (grant no. 81272167).

\section{References}

1. Assessment of fracture risk and its application to screening for postmenopausal osteoporosis. Report of a WHO study group. World Health Organ Tech Rep Ser 843: 1-129, 1994.

2. Bhutani G and Gupta MC: Emerging therapies for the treatment of osteoporosis. J Midlife Health 4: 147-152, 2013.

3. Han S, Chen JJ and Chen JZ: Latest progress in the identification of novel synthetic ligands for the cannabinoid CB2 receptor. Mini Rev Med Chem 14: 426-443, 2014.

4. Vasileiou I, Fotopoulou G, Matzourani M, Patsouris E and Theocharis S: Evidence for the involvement of cannabinoid receptors' polymorphisms in the pathophysiology of human diseases. Expert Opin Ther Targets 17: 363-377, 2013.

5. Yang P, Wang L and Xie XQ: Latest advances in novel cannabinoid CB (2) ligands for drug abuse and their therapeutic potential. Future Med Chem 4: 187-204, 2012.

6. Idris AI, van't Hof RJ, Greig IR, Ridge SA, Baker D, Ross RA and Ralston SH: Regulation of bone mass, bone loss and osteoclast activity by cannabinoid receptors. Nat Med 11: 774-779, 2005.

7. Ofek O, Karsak M, Leclerc N, Fogel M, Frenkel B, Wright K, Tam J, Attar-Namdar M, Kram V, Shohami E, et al: Peripheral cannabinoid receptor, CB2, regulates bone mass. Proc Natl Acad Sci USA 103: 696-701, 2006.

8. Sophocleous A, Landao-Bassonga E, Van't Hof R, Ralston S and Idris $A$ : The type 2 cannabinoid receptors $\left(\mathrm{CB}_{2}\right)$ protects against age-related osteoporosis by affecting bone formation and $\mathrm{CB}_{2}$ agonists exhibit anabolic activity in vivo. Bone 44: S219, 2009.

9. Sun YX, Xu AH, Yang Y, Zhang JX and Yu AW: Activation of cannabinoid receptor 2 enhances osteogenic differentiation of bone marrow derived mesenchymal stem cells. Biomed Res Int 2015: 874982, 2015.

10. Idris AI, Sophocleous A, Landao-Bassonga E, Canals M, Milligan G, Baker D, van't Hof RJ and Ralston SH: Cannabinoid receptor type 1 protects against age-related osteoporosis by regulating osteoblast and adipocyte differentiation in marrow stromal cells. Cell Metab 10: 139-147, 2009.

11. Fernandes H, Dechering K, Van Someren E, Steeghs I, Apotheker M, Leusink A, Bank R, Janeczek K, Van Blitterswijk C and de Boer J: The role of collagen crosslinking in differentiation of human mesenchymal stem cells and MC3T3-E1 cells. Tissue Eng Part A 15: 3857-3867, 2009.

12. Wu L, Wu Y, Lin Y, Jing W, Nie X, Qiao J, Liu L, Tang W and Tian W: Osteogenic differentiation of adipose derived stem cells promoted by overexpression of osterix. Mol Cell Biochem 301: 83-92, 2007.

13. Livak KJ and Schmittgen TD: Analysis of relative gene expression data using real-time quantitative PCR and the 2(-Delta Delta C(T)) method. Methods 25: 402-408, 2001.

14. Giusti A and Bianchi G: Treatment of primary osteoporosis in men. Clin Interv Aging 10: 105-115, 2014.

15. Maruotti N, Corrado A, Neve A and Cantatore FP: Bisphosphonates: Effects on osteoblast. Eur J Clin Pharmacol 68: 1013-1018, 2012.

16. Russell RG: Bisphosphonates: The first 40 years. Bone 49: 2-19, 2011. 
17. Rudic JS, Poropat G, Krstic MN, Bjelakovic G and Gluud C: Hormone replacement for osteoporosis in women with primary biliary cirrhosis. Cochrane Database Syst Rev: CD009146, 2011.

18. Montagnani A and Gonnelli S: Antidiabetic therapy effects on bone metabolism and fracture risk. Diabetes Obes Metab 15: 784-791, 2013.

19. Lewiecki EM: Current and emerging pharmacologic therapies for the management of postmenopausal osteoporosis. J Womens Health (Larchmt) 18: 1615-1626, 2009.

20. Caplan AI: Mesenchymal stem cells. J Orthop Res 9: 641-650, 1991.

21. Dore-Duffy P: Pericytes: Pluripotent cells of the blood brain barrier. Curr Pharm Des 14: 1581-1593, 2008.

22. Sousa-Victor P, Garcia-Prat L, Serrano AL, Perdiguero E and Muñoz-Cánoves P: Muscle stem cell aging: Regulation and rejuvenation. Trends Endocrinol Metab 26: 287-296, 2015.
23. Carrió E and Suelves M: DNA methylation dynamics in muscle development and disease. Front Aging Neurosci 7: 19, 2015.

24. Ramirez SH, Haskó J, Skuba A, Fan S, Dykstra H, McCormick R, Reichenbach N, Krizbai I, Mahadevan A, Zhang M, et al: Activation of cannabinoid receptor 2 attenuates leukocyte-endothelial cell interactions and blood-brain barrier dysfunction under inflammatory conditions. J Neurosci 32: 4004-4016, 2012.

25. Adhikary S, Li H, Heller J, Skarica M, Zhang M, Ganea D and Tuma RF: Modulation of inflammatory responses by a cannabinoid-2-selective agonist after spinal cord injury. J Neurotrauma 28: 2417-2427, 2011. 